\title{
The Somatization of Depression in Native Pakistani Women
}

\author{
Rubeena H. Mian M.D. \\ University of Illinois College of Medicine
}

Linda S. Grossman Ph.D.

Adult Outpatient Psychiatric Services; Department of Psychiatry at the University of Illinois College of Medicine

Follow this and additional works at: https://jdc.jefferson.edu/jeffjpsychiatry

Part of the Psychiatry Commons

Let us know how access to this document benefits you

\section{Recommended Citation}

Mian, Rubeena H. M.D. and Grossman, Linda S. Ph.D. (1998) "The Somatization of Depression in Native Pakistani Women," Jefferson Journal of Psychiatry. Vol. 14 : Iss. 1 , Article 4.

DOI: https://doi.org/10.29046/JJP.014.1.003

Available at: https://jdc.jefferson.edu/jeffjpsychiatry/vol14/iss1/4

This Article is brought to you for free and open access by the Jefferson Digital Commons. The Jefferson Digital Commons is a service of Thomas Jefferson University's Center for Teaching and Learning (CTL). The Commons is a showcase for Jefferson books and journals, peer-reviewed scholarly publications, unique historical collections from the University archives, and teaching tools. The Jefferson Digital Commons allows researchers and interested readers anywhere in the world to learn about and keep up to date with Jefferson scholarship. This article has been accepted for inclusion in Jefferson Journal of Psychiatry by an authorized administrator of the Jefferson Digital Commons. For more information, please contact: JeffersonDigitalCommons@jefferson.edu. 


\title{
The Somatization of Depression in Native Pakistani Women
}

\author{
Rubeena H. Mian, M.D. and Linda S. Grossman, Ph.D.
}

\begin{abstract}
Previous studies have pointed out the comorbidity of somatization and depression among non-Western psychiatric patients. We studied the somatization of depression in Pakistani women. Two groups were studied: inpatients with DSM-III-R diagnoses of major depression and a comparison sample of women living in the community without psychiatric diagnoses. Both groups were administered a rating scale for somatization. Results indicated that patients reported significantly more somatic symptoms than nonpatients. These findings support the concept that somatization may be a cultural mode of mental distress among Pakistani women, as has been observed for other non-Western populations.
\end{abstract}

\section{INTRODUCTION}

There has been an increased discussion in the recent psychiatric literature of the phenomenon of "somatization." Somatization can be defined as the expression of psychological and/or social distress through physical or somatic symptoms. The etiology of somatization is multifactorial incorporating genetics, personality, developmental learning, and social and cultural factors (1). There is strong support for the notion that the tendency to somatize depression is affected by culture (2). Studies from India, China, Mexico and Africa have noted that patients from developing countries "somatize" depression, whereas patients from the western world "psychologize" depression (3). Thus in most non-western cultures (as well as in many western cultures), individuals are not encouraged to express psychological distress verbally. In some cultures, this pattern has evolved because expression of emotional problems incurs a stigma which the expression of physical problems does not. Indeed in some cultures, negative affective states like depression and anxiety connote moral weakness (4).

The cultural component of somatization can be viewed broadly to include individuals' past learned experience with affect, as well as current feedback regarding acceptable ways of expressing affect. Influencing factors in the expression of affect

Send reprint requests and correspondences to: Linda S. Grossman, Ph.D., Department of Psychiatry, University of Illinois College of Medicine, 912 S. Wood Street (M/C 913), Chicago, Illinois 60612 
can include the values of one's family and social network, one's level of instrumental functioning, the availability of medical or psychological treatment, and the sociopolitical system to which one is exposed. There may also be a cultural component to the degree to which individuals are socially encouraged to develop the verbal and cognitive skills required to describe distress in psychological terms. Even if they have developed such skills, individuals may be differentially acculturated to develop psychological defenses and coping mechanisms which may impede expression of emotional distress. Cultures which discourage expression of affect may produce patients who have either not learned to perceive their negative emotions, or who are influenced by their support system to view depression as physical illness. Thus patients with major depression may selectively focus on their physical symptoms, and may experience their depression primarily in terms of somatic symptoms $(4,5)$.

Another aspect influencing expression of psychological difficulties as physical symptoms is gender. Studies of inpatients have indicated significant gender differences in somatization, suggesting women tend to internalize and somatize the experience of clinical depression, while men tend to externalize and act out $(6,7)$. The current paper focuses on somatization and depression in Pakistani women, a group for whom there have been to date no studies in this area.

\section{METHODS}

This study was conducted at the Mayo Hospital's Department of Psychiatry in Lahore, Pakistan. Two groups of Pakistani women were assessed and compared by Students t-tests. The first group comprised 30 female psychiatric inpatients who met DSM-III-R criteria for major depression, based on chart review and clinical interview. This group included all such patients admitted during a one-week period in January, 1994. The other group comprised 30 women selected at random from a local neighborhood in Lahore and matched for age, income, and education. This comparison group had no current or past psychiatric diagnosis. There was no difference between the two groups in age $(\mathrm{t}=1.25, \mathrm{p}>20)$. The mean age of the sample was 36.8 (士4.03). All subjects were working class urban individuals, with less than a high school education. Informed consent was obtained from all subjects.

All subjects were administered the Lahore Somatic Inventory (LSI, 4). We selected the LSI because it was adapted from the Bradford Somatic Inventory (BSI) which was specifically normed on Pakistani patients, as well as British patients, and has been shown successfully to assess $90 \%$ of somatic symptoms in these groups (3). This inventory is a 21 -item self-rated scale adapted from the 46 -item BSI $(3,8,9)$. It was originally based on the authors' systematic search of case notes for symptoms in Pakistan and Great Britain, and was further tested against case notes of psychiatric patients from other parts of the Indo-Pak subcontinent. The BSI was conceived bilingually in Urdu, the native language of Pakistan, and in English. It was written so that cultural nuances and idioms were readily comprehensible in both languages. A 
test of linguistic equivalence of the Urdu and English versions conducted on a sample of bilingual subjects produced satisfactory concordance for the two languages (3). In the current study, we administered the LSI in the Urdu version. The instrument taps a variety of somatic symptoms including headaches, gastrointestinal distress, tension in neck, shoulders or head, dry mouth, chest or heart discomfort, fatigue, sweating, trembling, and cold hands/feet. It assesses the presence of these symptoms during the past month, subdividing them according to whether patients experienced them less than or more than fifteen days out of the month.

\section{RESULTS}

The data from the LSI were analyzed in two ways. First the groups were compared on the total number of symptoms they reported, and then the severity of these symptoms as measured by a three-point scale was compared. The results indicated that the inpatient group reported significantly more somatic symptoms $(\mathrm{t}=10.18, \mathrm{df}=58, \mathrm{p}<.0001)$ than the nonpatient group. The mean number of symptoms reported by the patient sample was $11.83( \pm 4.8)$, and the mean reported by the nonpatient sample was $2.4( \pm 1.6)$. We obtained a similar significant difference $(\mathrm{t}=10.86, \mathrm{df}=58, \mathrm{p}<.0001)$ when we compared the two groups on severity of symptoms. The patients' mean severity score was $21.9( \pm 9.7)$, and the nonpatients' score was $2.4( \pm 1.6)$.

\section{DISCUSSION}

In order to achieve improvements in diagnosis and mental health care, psychiatrists need to be increasingly aware of transcultural issues. Immigrant minorities make up a large percentage of the United States population and comprise a factor which cuts across all psychiatric research. Therefore a wider acknowledgment of cultural differences will increase awareness and lead ultimately to improvement in mental health care.

A high incidence of somatization disorder diagnosed in outpatient clinics has been noted in the literature, especially in primary care settings (10,11). Many patients attending hospital medical outpatient clinics in Pakistan complain of somatic symptoms for which no physical cause is found (8). It is possible that many of these patients may have underlying depression or anxiety. In our study, depressed Pakistani women were found to have more somatic symptoms than non-depressed matched control subjects.

While the relationships among somatization, depression, and anxiety have not received widespread research attention in Pakistan, one study found that as many as one-third of a sample of Pakistani immigrants attending medical clinics in Great 
Britain show some degree of psychiatric disturbance (9). Although the BSI does not diagnose psychiatric disorders, it is helpful in identifying patients with somatic symptoms. Therefore, use of rating scales such as the BSI may help clinicians to avoid expensive and time-consuming medical diagnostic investigations and inappropriate medical treatment, and may lead to more appropriate psychiatric interventions.

The current study was limited in that it was an open study of female patients with major depression and those with no psychiatric disorders, and did not take into account the potential influence of treatment, physical illnesses, or the menstrual cycle. However, the findings from this study suggest that somatic complaints can be used as diagnostic clues in the assessment of female Pakistani patients to heighten the clinician's sensitivity to the possibility of an underlying diagnosis of depression. Our data suggest that Pakistani working class women with less than high school educations may express their emotional distress primarily in somatic symptoms. This finding is in accord with previous data (9) indicating that patients who express psychological distress through physical symptoms tend to be undereducated or to come from cultural groups where emotional distress is expressed through physical symptoms. An interesting direction for future research on Pakistani immigrants to the United States who seek psychiatric or medical attention, would be to compare native populations with immigrants, to provide more comprehensive information concerning the impact of acculturation on patients' tendency to express emotional distress and on their tendency to somatize depression and anxiety.

\section{REFERENCES}

1. Lipowski ZJ: Somatization. The concept and its clinical application. Am J Psychiatry 1988; 145:1358-1368.

2. Teja JS, Narang RL, Aggarwal AK: Depression across cultures. Br J Psychiatry 1971; 119:253-60.

3. Mumford DB, Bavington JT, Bhatnagar KS et al: The Bradford Somatic Inventory, a multi-ethnic inventory of somatic symptoms reported by anxious and depressed patients in Britain and the Indo-Pakistan subcontinent. Br J Psychiatry 1991; 158:379-3862.

4. Katon W, Kleinman A, Rosen G: Depression and somatization: A review. Am J Med 1982; 72:127-135.

5. Kellner R: Somatization theories and research.J Nerv Ment Dis 1990; 178:150-159.

6. Golding JM, Aneshensel CS: J Clin Psychology 1991; 47:61-74.

7. Velasquez RJ, Callahan WJ: MMPI differences among Mexican-American male and female psychiatric inpatients. Psychol Rep 1991; 68:123-127.

8. Mumford DB, Tareen MR, Bhatt MAZ, et al: An investigation of "functional" somatic symptoms among patients attending hospital medical clinics in Pakistan: II. Using somatic symptoms to identify patients with psychiatric disorders. J Psychosom Res 1991; 35:257-264.

9. Mumford DB, Tareen IAK, Bajwa MR et al.; An investigation of 'functional' somatic symptoms among patients attending hospital medical clinics in Pakistan: I. Characteristics of 'non-organic' patients. J Psychosom Res 1991; 35:245-255. 
Appendix 1

Lahore Somatic Index

Name

Sex

Age

Study No

We would like to know if you have had any body symptom over the past month. Please answer all the questions by checking the appropriate box. Remember that we want to know about the past month, not symptoms you have had before that. If is very important that you try to answer all the questions. Thank you very much for your cooperation.

"During the past month. .."

Present on Present on less than more than 15 days in 15 days in

1. Have you had severe headaches?

2. Have you had fluttering or a feeling of something moving in your stomach?

3. Have you had pain or tension in your neck and shoulders?

4. Have you had a feeling of constriction of your head, as if it was being gripped tightly from outside?

5. Have you felt pain in the chest or heart?

6. Has your mouth or throat felt dry?

7. Have you felt a lack of energy (weakness) much of the time?

8. Have you been sweating a lot?

9. Have you felt as if there was pressure or lightness on your chest or heart?

10. Has there been a choking sensation in your throat?

11. Have you felt aches or pains all over the body?

12. Have you been aware of palpitations (heart pounding)?

13. Have you been trembling or shaking?

14. Have you been passing urine more frequently?

15. Has your head felt heavy?

16. Have you been feeling tired, even when you are not working?

17. Have you had a feeling of pressure inside your head as if your head was going to burst?

18. Have you been troubled by constipation?

19. Has your heart felt weak or sinking?

20. Have you suffered from excessive wind (gas) or belching?

21. Have your hands or feet felt cold? 